Les textes complets des travaux, accompagnés d'un résumé et d'un curriculum vitae, doivent lui être soumis dans ce délai, en deux exemplaires. Un exemplaire des travaux primés restera propriété de la SPHN.

Ces textes peuvent être rédigés en français, allemand, italien ou anglais. Dans ces trois derniers cas le résumé sera traduit en français et comprendra une douzaine de pages, soit 4000 mots ou 20000 signes environ.

Le thème choisi pour le Prix en 1992 concerne l'Histoire de la chimie.

Une commission est chargée d'examiner les candidatures.

Le Président de la SPHN

Marc-Auguste Pictet (1752-1825) fut l'un des huit fondateurs de la Société de Physique et d'Histoire naturelle de Genève, en 1790. Il fut aussi, en 1796, l'un des créateurs de la revue littéraire et scientifique internationale «La Bibliothèque Britannique», ancêtre de l'actuelle revue «Archives des Sciences» éditée par la SPHN.

Il succéda à H. B. de Saussure dans la chaire de philosophie (naturelle) de l'Académie de Genève, puis à Jacques André Mallet dans celle d'astronomie assumant la direction de l'Observatoire de Genève pendant 30 ans. Membre de la Société Royale de Londres et de l'Académie des Sciences de Paris, il fut l'un des savants les plus en vue de son époque.

\title{
Eine Zuschrift
}

Entgegnung auf die Rezension von Cornelia Isler-Kerényi über das Buch von Hildegard Sobel, Hygieia. Die Göttin der Gesundheit, WB Darmstadt 1990 (Gesnerus 47, 1990, 394.f.)

Was der Verfasserin der Rezension offenbar vorschwebt, «eine wirkliche verehrungswürdige religiöse Gestalt zu entdecken», war nicht die Absicht meiner Abhandlung. Mein Anliegen war es, die archäologisch relevanten Objekte zu erfassen und daraus die Typologie der Hygieia und deren Wandlung im Verlaufe von acht Jahrhunderten zu beschreiben. Die Schriftquellen wurden berücksichtigt.

Wenn es auch einige literarische Belege für einen älteren selbständigen Kult der Hygieia gibt und auch eine Athena Hygieia des 5. Jh. vor Chr. bezeugt ist, tritt die Hygieia, die Kultgefährtin des Asklepios, erst gegen Ende des 5. Jh. vor Chr. in Erscheinung und entleiht ihre Ikonographie von der anderer Göttinnen. Auch der Paean des Ariphron stammt erst aus dem 4. Jh. vor Chr. Aus dem Aussehen, den Attributen und Beziehungen Hygieias neue Einsichten in ihr Wesen zu gewinnen, halte ich für gewagt, wenn man 
bedenkt, daß die archäologischen Objekte großenteils Kopien darstellen, die zudem häufig schlecht oder falsch restauriert sind. Selbst die Zuweisung der ursprünglichen Originale an berühmte Bildhauer der Antike ist fragwürdig. In jeder vordergründigen Ähnlichkeit eine «tiefere Affinität» zu suchen, wäre nicht beweisbar. C.Isler-Kerényi stört sich offenbar auch an dem Begriff der personifizierten Wunschvorstellung, der natürlich ein Hilfsbegriff ist, den man aber gebrauchen muß, bis ein besserer gefunden ist.

In der Zwischenzeit erschien der Lexikonartikel «Hygieia» im LIMC, V, I und II, Artemis-Verlag Zürich und München 1990, von Francis Croissant, dessen Ergebnisse sich mit meiner Abhandlung vergleichen lassen.

Hildegard Sobel

D-6509 Undenheim

\section{Communication de la rédaction}

Le comité de rédaction de Gesnerus avait prévu de publier à la fin de l'été 1991 le volume contenant les communications présentées au Symposium sur l'histoire de la médecine, de la santé publique et de la biologie en rapport avec la Suisse romande qui s'est tenu à Genève les 5 et 6 octobre 1990 dans le cadre de la réunion annuelle de la Société suisse d'histoire de la médecine et des sciences naturelles. Ce volume devait constituer la livraison 2/3 de l'année en cours. Mais l'ampleur des textes à publier a dépassé les prévisions et retardé la préparation du volume. Celui-ci paraîtra donc au début de 1992 et constituera la livraison $3 / 4$ du volume 48 .

Nous présentons donc ici selon le rythme habituel de parution de notre revue le deuxième cahier de l'année 1991 qui contient comme à l'ordinaire des contributions touchant à des domaines variés de l'histoire de la médecine et des sciences naturelles et nous présentons à nos lectrices et à nos lecteurs nos vœux pour d'heureuses fêtes de fin d'année. 